\title{
The usefulness of telemedicine devices in patients with severe heart failure with an implanted cardiac resynchronization therapy system during two years of observation
}

\author{
Marcin Wita' , Michał Orszulak', Krzysztof Szydło' ${ }^{1}$, Wojciech Wróbel' ${ }^{1}$ Artur Filipecki ${ }^{1}$, Karolina Simionescu², \\ Karol Sanecki², Urszula Uchwat ${ }^{3}$, Maciej Wybraniec' ${ }^{1}$, Zbigniew Tabor ${ }^{4}$, Krzysztof Gołba $^{2}$, Krystian Wita', \\ Katarzyna Mizia-Stec ${ }^{1}$ \\ ${ }^{1} 1^{\text {st }}$ Department of Cardiology, School of Medicine in Katowice, Medical University of Silesia, Katowice, Poland \\ 2Department of Electrocardiology and Heart Failure, S School of Medicine in Katowice, Medical University of Silesia, Katowice, Poland \\ ${ }^{3}$ Department of Neurology, Professor Leszek Giec, Upper-Silesian Heart Center, Medical University of Silesia, Katowice, Poland \\ ${ }^{4}$ Telemedycyna Polska SA, Poland
}

\author{
Correspondence to: \\ Marcin Wita, MD, \\ $1^{\text {st }}$ Department of Cardiology, \\ School of Medicine in Katowice, \\ Medical University of Silesia, \\ Ziołowa 45/47, 40-635 Katowice, \\ Poland, \\ phone: +48323598890 \\ e-mail:mwita@onet.pl \\ Copyright by the Author(s), 2022 \\ DOI: 10.33963/KP.a2021.0175 \\ Received: \\ July 12, 2021 \\ Accepted: \\ December 8, 2021 \\ Early publication date: \\ December 9, 2021
}

\section{A B S TRA C T}

Background: Heart failure (HF) remains a disease with a poor prognosis. Telemonitoring is a medical service aimed at remote monitoring of patients.

Aim: The study aimed to identify the clinical relevance of non-invasive telemonitoring devices in HF patients.

Methods: Sixty patients aged 66.1 (11) years, with left ventricular ejection fraction (LVEF) 26.3 (6.8)\% underwent cardiac resynchronization therapy (CRT) implantation. They were randomly allocated to the control (standard medical care) or study (standard medical care + telemonitoring device) groups. During the follow-up ( 24 months), the patients in the study group provided body mass and blood pressure, along with electrocardiogram on a daily basis. The data were transferred to the monitoring center and consulted with a cardiologist. Transthoracic echocardiography and a 6-minute walk test were performed before and 24 months after CRT implantation.

Results: During the two-year observation, the composite endpoint (death or HF hospitalization) occurred in 21 patients, more often in the control group (46.8\% vs. $21.4 \% ; P=0.026)$. In univariate analysis: the use of telemetry (hazard ratio [HR], $0.2 ; 95 \%$ confidence interval $[\mathrm{Cl}], 0.07-0.7 ; P=0.004$ ), the presence of coronary heart disease $(\mathrm{HR}, 41.4 ; 95 \% \mathrm{Cl}, 3.1-567.7 ; P=0.005)$, hypertension $(\mathrm{HR}$, $0.24 ; 95 \% \mathrm{Cl}, 0.07-0.90 ; P=0.035)$, and patient's body mass $(\mathrm{HR}, 0.36 ; 95 \% \mathrm{Cl}, 0.14-0.92 ; P=0.03)$ were related to the occurrence of the composite endpoint.

Conclusions: The use of a telemonitoring device in CRT recipients improved the prognosis in 2-year observation and contributed to the reduction of HF hospitalization.

Key words: cardiac resynchronization therapy, COVID-19, heart failure, telemedicine

\section{INTRODUCTION}

In the $21^{\text {st }}$ century, heart failure (HF) has become a disease of civilization. It is estimated that the disease occurs in $1 \%-2 \%$ of the adult population, reaching the value of about $20 \%$ in the population of patients over the age of 80. Ischemic heart disease increases the risk of HF in two-thirds of patients. Other causes of $\mathrm{HF}$ are arterial hypertension, viral infection, or alcohol abuse [1].

Nearly half of the patients diagnosed with HF die within 4 years, and in the group of patients with severe chronic HF, over $50 \%$ die within 1 year. Despite modern pharma- 
WHAT'S NEW?

The use of telemedicine care in the population of patients with severe heart failure undergoing cardiac resynchronization therapy reduces the occurrence of a composite endpoint in long-term observation. The use of telemetry decreases the risk of hospitalization for exacerbation of heart failure. Heart-failure patients can aid themselves with the use of telemedicine devices.

cotherapy, the annual mortality rate among HF patients is about $20 \%$ in people below 75 years and more than $40 \%$ in patients over 75 years [2].

One of the essential elements of the treatment of heart failure with reduced ejection fraction (HFrEF) is a procedure involving the use of implantable devices, especially cardiac resynchronization therapy (CRT). In a randomized study, the Multicenter Automatic Defibrillator Implantation Trial with Cardiac Resynchronisation Therapy (MADIT-CRT), device-based therapy has proven to reduce mortality and incidence of HF [3].

The telemonitoring application allows for the detection of symptoms and abnormal health parameters earlier than during a routine consultation. It may also reduce the need for frequent visits to healthcare facilities, thereby increasing the patient's quality of life. A detailed study of previous research projects conducted in the field of telemonitoring indicates a reduction in the number of hospitalizations for exacerbation of HF [4]. Therefore, the European Society of Cardiology (ESC) recommends the use of telemonitoring to improve the quality of care of patients with HF [5].

\section{Aim of the study}

The primary aim of the study was to identify the clinical relevance of telemedicine devices for the reduction of the composite endpoint defined as death or first-time emergency hospitalization for HF in patients with implanted CRT in long-term observation.

The secondary aim of the study was to determine the relevance of telemetry use in complex HF management for the improvement of the following clinical endpoints and parameters:

- distance of the six-minute walk test (6MWT);

- indices of left ventricular (LV) systolic function expressed as left ventricular ejection fraction (LVEF);

- and left ventricular global longitudinal strain (LV GLS) in a two-year observation.

\section{METHODS}

Consecutive patients with HFrEF eligible for cardiac resynchronization therapy (CRTP/CRTD) fulfilling the criteria of class I or Ila recommendations according to the Cardiac Pacing and Cardiac Resynchronisation Therapy Guidelines of 2013 were prospectively enrolled in the study between 2014 and 2017.

Before CRT implantation, demographic data were obtained. Considering the etiology of HF, laboratory tests including the level of the $\mathrm{N}$-terminal pro-brain natriuretic pep- tide (NT-proBNP), echocardiography, and a 6MWT were performed.

The two-year follow-up assessment included the same set of investigations.

Patients were randomly divided (1:1) into two groups (Figure 1): the first comprised patients receiving standard medical care (the control group), and the second involved patients who additionally were monitored with the use of telemedical devices (the study group).

\section{Telemedicine device}

At discharge, after a short training, patients from the study group received a telemonitoring set: a blood pressure (BP) monitor, a 3-lead electrocardiogram (ECG) recorder, a weighing machine, and a personal tablet. Each device was connected to the tablet with an application. None of the patients received a remote monitoring system of implantable devices.

Patients were asked to perform the measurements of body weight, BP and to evaluate their subjective well-being once a day. Patients were asked to register ECG every week. To evaluate the biventricular stimulation in 3-lead ECG, each patient had an ECG recording on the day of discharge, which was used as a base to compare with further ECGs.

Data were recorded by the patients themselves and transmitted with the use of a tablet to the monitoring center. Patients were also offered the possibility of medical teleconsultation.

To individualize care in our study, we were guided by the parameter trends in the results from the previous week for each patient separately.

All medical devices used in the project were CE marked and provided by Meditel Company, Poland. Throughout the entire study, patients were under the care of the Cardiological Outpatient Clinic in Katowice-Ochojec. During the planned follow-up appointments, data from the devices were verified. All patients were treated with optimal pharmacotherapy for HF and co-morbidities. All patients were informed about the study and signed a written consent to participate in it. The study protocol was approved by the local bioethical committee.

\section{Laboratory test}

NT-proBNP was determined on the first day of hospitalization and during a check-up after 24 months by means of the electrochemiluminescence method using the ELECSYS 1010 (Roche Diagnostics, Basel, Switzerland) analyzer. 


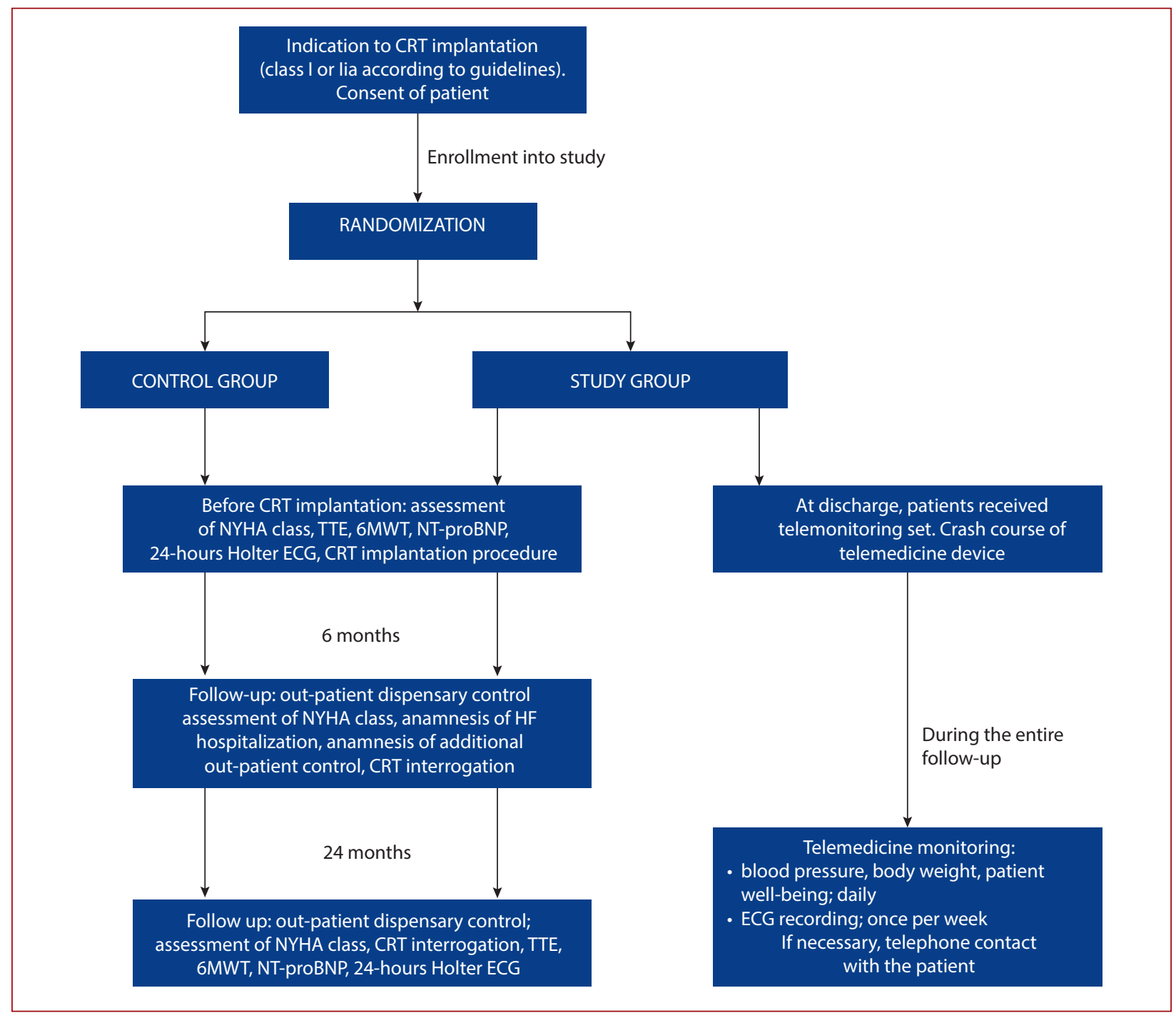

Figure 1. Work plan — flow-chart of the study

Abbreviations: 6MWT, distance of the six-minute walk test; CRT, cardiac resynchronization therapy; ECG, electrocardiogram; HF, heart failure; NT-proBNP, N-terminal pro-brain natriuretic peptide; NYHA, New York Heart Association; TTE, transthoracic echocardiographic examination

\section{Six-minute walk test}

$6 \mathrm{MWT}$ was performed on the day of admission and after 24 months during the outpatient follow-up appointment. The improvement of functional exercise capacity was defined as a relative increase of the distance walked by at least $10 \%$.

\section{Echocardiography}

A two-dimensional resting echocardiographic examination was performed upon admission to the hospital and after two years. Left ventricular end-diastolic volume (LVEDV) and left ventricular end-systolic volume (LVESV) were determined using Simpson's method; LVEF was calculated as a percentage of change in the volume of the left ventricle between its diastole and systole, as the arithmetic mean from 3 consecutive measurements. LVEF improvement in the long-term observation was defined as a relative increase of at least $5 \%$.
An echocardiographic examination of myocardial strain (systolic time interval [STI]) was performed directly after a traditional echocardiographic examination. The mean peak value of all 16 segments of the left ventricle (GLS, global longitudinal strain) was evaluated. GLS improvement in the long-term observation was defined also as an increase of at least $5 \%$.

\section{Long-term observation}

The primary composite endpoint was defined as all-cause death or first-time emergency hospitalization for decompensated HF. The secondary endpoint comprised all components of the primary endpoint, as well as:

- improvement of $6 \mathrm{MWT}$ distance $>10 \%$;

- improvement of LVEF > $5 \%$;

- Improvement of GLS $>5 \%$.

Every patient (the study and control groups) underwent a two-year follow-up with regular outpatient 
Table 1. Baseline characteristics of the general population

\begin{tabular}{|c|c|c|c|c|}
\hline & $\begin{array}{l}\text { General population } \\
\qquad(n=60)\end{array}$ & $\begin{array}{l}\text { Study group } \\
\qquad(n=28)\end{array}$ & $\begin{array}{l}\text { Control group } \\
(n=32)\end{array}$ & $P$-value \\
\hline Female sex, n (\%) & $13(21.7)$ & $5(17.9)$ & $8(25)$ & 0.5 \\
\hline Age, years, mean (SD) & $66.1(10.5)$ & $65.1(11.7)$ & $66.9(9.3)$ & 0.48 \\
\hline Ischemic etiology of HF, n (\%) & $29(48.3)$ & $13(46.4)$ & $16(50)$ & 0.78 \\
\hline NYHA class, median (IQR) & $3(2-3)$ & $2(2-3)$ & $3(2-3)$ & 0.18 \\
\hline LBBB, n (\%) & $41(68.3)$ & $18(64.3)$ & $23(71.9)$ & 0.53 \\
\hline Diabetes, n (\%) & $15(25)$ & $3(10.7)$ & $12(37.5)$ & 0.02 \\
\hline Arterial hypertension, $\mathrm{n}(\%)$ & $47(78.3)$ & $21(75)$ & $26(81.2)$ & 0.34 \\
\hline GFR, ml/min, median (IQR) & $64.59(51.8-90.6)$ & $67.8(59.3-88.9)$ & $63.5(44.8-94.9)$ & 0.61 \\
\hline Active smoking, n (\%) & $29(48)$ & $12(42.9)$ & $17(53.1)$ & 0.43 \\
\hline Stroke/TIA, n (\%) & $7(11.7)$ & $2(7.1)$ & $5(15.6)$ & 0.31 \\
\hline QRS width, ms, median (IQR) & $160(160-180)$ & $162.5(155-180)$ & $160(160-180)$ & 0.98 \\
\hline Permanent AF, n (\%) & $10(16.7)$ & $4(14)$ & $6(18.7)$ & 0.58 \\
\hline 6MWT, m, mean (SD) & $336(118)$ & $359.6(106)$ & $315.4(126)$ & 0.15 \\
\hline NT-proBNP, pg/ml, median (IQR) & $1860(1020-3410)$ & 1709 (996-2393) & $2011(1054-3912)$ & 0.20 \\
\hline CRT-D, n (\%) & $58(97)$ & $27(96)$ & $31(97)$ & 0.89 \\
\hline CRT-P, n (\%) & $2(3)$ & $1(4)$ & $1(3)$ & 0.86 \\
\hline
\end{tabular}

Abbreviations: AF, atrial fibrillation; BSA, body surface area; GFR, glomerular filtration rate; LBBB, left bundle branch block; TIA, transient ischemic attack; other — see Figure 1

appointments at $6,12,18$, and 24 months. The primary composite endpoint was evaluated on every outpatient visit, while in the case of loss of contact with the patient, data from medical records and National Healthcare Provider were obtained to establish the exact date and cause of the event. The follow-up time was 24 months. The secondary endpoints of improvement of 6MWT and echocardiographic parameters were established at a 24-month follow-up visit.

\section{Statistical analysis}

Statistical analysis was performed using Statistica 10 software (BCO Software Inc., Palo Alto, CA, USA). Distribution was verified using the Shapiro-Wilk test. Continuous variables are expressed as the mean (standard deviation [SD]) in the case of a normally distributed variable, whereas non-normally distributed variables were expressed as the median and interquartile range (IQR). Qualitative parameters were expressed as crude values and percentages. Categorical variables were tested using X2 statistics. The comparison of non-paired continuous variables was performed using the Mann-Whitney $U$ test or Student's t-test, while paired variables were verified using the Wilcoxon signed-rank test or paired-sample Student's t-test. Multivariable logistic regression analysis was performed using a stepwise approach to evaluate the predictors of $6 \mathrm{MWT}, \mathrm{GLS}$, and LVEF improvement. The Cox proportional hazards model was used to identify independent predictors of composite end point occurrence. Kaplan-Meier survival curves were calculated to compare freedom from the composite endpoint in relation to the use of telemedicine devices. The difference between survival curves was compared using the logrank test. The $P$-value $<0.05$ was regarded as statistically significant throughout the study analyses.

\section{RESULTS}

Initially, 63 patients were eligible to take part in the study. Out of the 31 patients initially recruited to the study group, 1 patient died during hospitalization, 2 withdrew their consent to participate in the study due to the technical limitations of the Internet at their place of residence. Not all the data concerning the excluded patients were analyzed. Demographic, clinical, and laboratory data of the study population depending on group allocation are presented in Table 1.

The examined groups differed only in terms of the prevalence of diabetes, which was more frequent in the control group (37.5\% vs. $10.7 \% ; P=0.02)$. There was no statistical difference in echocardiographic parameters between the examined groups (Table 2).

The patients were treated in accordance with the ESC recommendations, and the applied pharmacotherapy did not differ between the study groups, except for more frequent use of beta-blockers in the control group (100\% vs. $85.7 \%$; $P=0.03$; Supplementary material, Table S1).

On admission, the median NT-proBNP value for the whole population was $1860(1020-3410) \mathrm{ng} / \mathrm{ml}$ and decreased to 771 (440-2113) $\mathrm{ng} / \mathrm{ml}(P=0.01)$. A significant decrease in the median NT-proBNP value, from 1709 (996-2393) ng/ml to 553 (292-1256) ng/ml ( $P=0.002)$, occurred in the study group. The difference in the control group was not significant: $2011(1054-3912)$ ng/ml vs. 1560 (583-2671) $\mathrm{ng} / \mathrm{ml}(P=0.48)$.

A similar percentage of biventricular stimulation was demonstrated for both groups in the longterm follow-up (96.8\% [3] vs. 95.4\% [2]; $P=0.85$ ). The occurrence of ICD interventions was also similar: $25 \%$ of patients in the study group and $28 \%$ in the control group $(P=0.7)$.

There were 55 consultations in the study group during the follow-up. Most of them (18 phone calls) concerned the 
Table 2. Echocardiographic data

\begin{tabular}{|c|c|c|c|c|}
\hline & $\begin{array}{l}\text { General population } \\
\qquad(n=60)\end{array}$ & $\begin{array}{l}\text { Study group } \\
\qquad(n=28)\end{array}$ & $\begin{array}{l}\text { Control group } \\
(n=32)\end{array}$ & P-value \\
\hline LA area, $\mathrm{mm}^{2}$ mean (SD) & $29.4(8.7)$ & $28.8(9.1)$ & $29.9(8.5)$ & 0.59 \\
\hline LVEDV index, $\mathrm{ml} / \mathrm{m}^{2}$, mean (SD) & $125.7(40.7)$ & $133.7(50.5)$ & $118.7(28.7)$ & 0.16 \\
\hline LVESV index, $\mathrm{ml} / \mathrm{m}^{2}$, mean (SD) & $93.4(35.7)$ & $98.1(44.7)$ & $89.2(25.7)$ & 0.34 \\
\hline LVEF, \%, mean (SD) & $26.3(6.8)$ & $26.6(7)$ & $26.1(6.7)$ & 0.78 \\
\hline GLS, \%, mean (SD) & $-6.8(3.9)$ & $-6.7(4.9)$ & $-6.87(3.1)$ & 0.89 \\
\hline GCS, \%, mean (SD) & $-6.9(4.9)$ & $-7.2(5.5)$ & $-6.6(4.5)$ & 0.64 \\
\hline $\mathrm{GR}, \%$, mean $(\mathrm{SD})$ & $12.6(14)$ & $13.6(12.8)$ & $11.7(15.1)$ & 0.62 \\
\hline
\end{tabular}

Abbreviations: LA, left atrium; LVEDV, left ventricular end-diastolic volume; LVESV, left ventricular endsystolic volume; LVEF, left ventricular ejection fraction; GLS, global longitudinal strain; GCS, global circumferential strain; GRS, global radial strain

Table 3. Differences in the six-minute walk test (6MWT) and left ventricular ejection fraction (LVEF)

\begin{tabular}{|c|c|c|c|c|}
\hline \multicolumn{5}{|c|}{ 6MWT } \\
\hline & Before implantation CRT & After 2-year observation & $\triangle 6 M W T$ & P-value \\
\hline Study group, m, mean (SD) & $359.6(106)$ & $453(120)$ & $86.5(88.4)$ & 0.003 \\
\hline Control group, $\mathrm{m}$, mean (SD) & 315 (126) & 325 (176) & $29.4(129.4)$ & 0.48 \\
\hline \multicolumn{5}{|c|}{ LVEF } \\
\hline & Before implantation CRT & After 2-year observation & $\triangle L V E F$ & $P$-value \\
\hline Study group, $\%$, mean (SD) & $26.6(7)$ & $34.8(11.1)$ & $8.4(9.1)$ & 0.002 \\
\hline Control group, $\%$, mean (SD) & $26.1(6.7)$ & $35.9(13.2)$ & $9.8(12.1)$ & 0.005 \\
\hline
\end{tabular}

Abbreviations: see Tables 1 and 2

exacerbation of dyspnea. Apart from that, 14 consultations were arranged because of abnormal ECGs, 12 consultations due to an increase in body weight, and 11 on account of hyper- or hypotension. Due to these consultations, 29 additional outpatient visits were made to modify therapy. In addition, 13 scheduled hospitalizations were performed, including 3 for coronary angiography, 1 for endocarditis, and 3 for electrical cardioversion. Moreover, 6 hospitalizations were organized for intravenous pharmacotherapy.

Of the 26 remaining phone consultations, pharmacotherapy with diuretics was intensified 15 times, hypertensive therapy was modified 5 times, and beta-blocker therapy was modified 5 times. As pneumonia progressed, the patient was asked to increase hydration because of hypotension.

In the control group, 9 scheduled hospitalizations were organized, five of them to intensify therapy, 3 related to electrical cardioversion, and 3 for coronary angiography.

\section{CLINICAL ENDPOINTS}

\section{Six-minute walk test}

The baseline mean distance of the 6MWT in the whole population was 336 (118) $\mathrm{m}$ and reached 389.4 (162) $\mathrm{m}$ during the follow-up $(P=0.05)$. In the study group, the mean distance was 359.6 (106) $\mathrm{m}$ and reached 453 (120) $m$ in the 2-year observation period. In the control group, the results were 315 (126) $\mathrm{m}$ and 325 (176) $\mathrm{m}(P=0.004)$, respectively (Table 3 ).

Therefore, the mean distance of 6MWT for the study population generally increased by 58.5 (112.5) $\mathrm{m}$, reach-
Table 4. Multivariable logistic regression analysis for 6MWT improvement

\begin{tabular}{l|c|c} 
& OR (95\% Cl) & P-value \\
\hline Telemonitoring (yes) & $9.58(2.31-39.75)$ & 0.005 \\
ACE-I (yes) & $3.86(1.07-13.89)$ & 0.04 \\
NT-proBNP (as continuous variables), & $0.99(0.08-0.99)$ & 0.005 \\
$\mathrm{pg} / \mathrm{ml}$ & & \\
LA vol. (as continuous variables) & $0.88(0.80-0.98)$ & 0.01 \\
Tricuspid valve regurgitation (yes), $\mathrm{ml}$ & $0.51(0.27-0.97)$ & 0.003 \\
\hline
\end{tabular}

Abbreviations: ACE-I, angiotensin-converting enzyme inhibitor; $\mathrm{Cl}$, confidence interval; LA vol. left atrium volume; OR, odds ratio; other — see Table 1

ing an increase of 86.5 (88.4) $\mathrm{m}$ in the study group, and an increase of 29.4 (129.4) $\mathrm{m}$ in the control group, and there was a significant tendency $(P=0.07)$. Based on the adopted criteria, an improvement in 6MWT was observed in 34 patients, of whom 22 (85\%) were in the study group and $12(44 \%)$ in the control group ( $P=0.004)$. The predictors of 6MWT improvement were analyzed using the multivariable logistic regression analysis (Table 4).

\section{Echocardiographic indices of $L V$ systolic function}

The baseline LVEF in the whole population was $26.3 \%$ (6.8) and increased to $35.4 \%$ (12.1) after the 2-year observation period $(P<0.001)$. In the study group, LVEF increased from $26.6 \%(7)$ to $34.8 \%(11)(P=0.002)$, like in the control group (26.1\% [6.7] vs. 35.9\% [13.2] ( $P=0.005)$ (Table 3).

The mean $\triangle$ LVEF value was $9.2 \%$ (10.7) in the whole population. It was $8.4 \%(9.1)$ in the study group and $9.8 \%$ (12.1) in the control group $(P=0.6)$.

Based on the adopted criteria, an improvement of LVEF was observed in 41 patients, 21 (81\%) patients in the 
Table 5. Multivariable logistic regression analysis for LVEF improvement

\begin{tabular}{l|c|c} 
& OR (95\% Cl) & P-value \\
\hline Telemonitoring (yes) & $1.57(1.35-6.39)$ & 0.007 \\
Active smoking (yes) & $0.15(0.03-0.78)$ & 0.01 \\
MRA (yes) & $5.71(2.95-34.24)$ & 0.04 \\
NT-proBNP (as continuous variables), & $0.99(0.99-1.00)$ & 0.001 \\
pg/ml & & \\
Tricuspid valve regurgitation (yes) & $0.41(0.21-0.84)$ & 0.001 \\
\hline
\end{tabular}

Abbreviations: MRA, mineralocorticoid receptor antagonist; other — see Tables 1 and 4

study group, and $20(74 \%)$ in the control group ( $P=0.56)$. The predictors of LVEF improvement were analyzed using multivariable logistic regression (Table 5).

The mean of GLS in the whole population was $-6.8 \%$ (3.9) and decreased to $-10 \%$ (4.3) after the 2-year observation $(P=0.58)$. In the study group, GLS decreased from $-6.71 \%(4.9)$ to $-9.8 \%(4.5)(P=0.15)$, like in the control group $(-6.87 \%$ [3.1] vs. 9.4\% [4.1]; $P=0.2)$. There were no independent predictors of GLS improvement in the multivariable logistic regression analysis.

\section{Primary composite endpoint}

The median clinical observation time was 720 (30-750) days. The composite endpoint occurred in 21 patients (2 deaths, 19 first-time emergency hospitalizations for acute HF), more often in the control group: $46.8 \%$ vs. $21.4 \%$ $(P=0.03)$.

The hospitalization occurred in 19 patients, 5 in the study group $(P=0.03)$. Fatal cases caused by exacerbation of HF occurred in both groups.

Of all the assessed data, telemetry (hazard ratio [HR], $0.2 ; 95 \%$ confidence interval $[\mathrm{Cl}], 0.07-0.7 ; P=0.004)$, coronary artery disease $(\mathrm{HR}, 41.4 ; 95 \% \mathrm{Cl}, 3.1-567.7 ; P=0.005)$, arterial hypertension ( $\mathrm{HR}, 0.24 ; 95 \% \mathrm{Cl}, 0.07-0.90 ; P=0.035)$, and patient's body mass ( $\mathrm{HR}, 0.36 ; 95 \% \mathrm{Cl}, 0.14-0.92$; $P=0.033$ ) were related to the occurrence of the composite endpoint in univariate analysis.

In the multivariable Cox proportional hazard regression analysis, telemetry $(\mathrm{HR}, 0.3 ; 95 \% \mathrm{Cl}, 0.11-0.91 ; P=0.03)$, coronary artery disease $(\mathrm{HR}, 11.8 ; 95 \% \mathrm{Cl}, 1.56-89.5$; $P=0.01)$, and LV GLS values (HR, 0.02; $95 \% \mathrm{Cl}, 1.02-1.31$; $P=0.02$ ) predict the occurrence of the composite endpoint $(P=0.001)$.

Figures 2 and 3 present the analysis of freedom from the occurrence of the composite endpoint and hospitalization for acute $\mathrm{HF}$ in relation to the use of telemedicine devices in the long-term observation.

\section{DISCUSSION}

This randomized prospective study was designed to determine the real impact of telemedicine on the occurrence of the endpoints in the long-term observation. The main

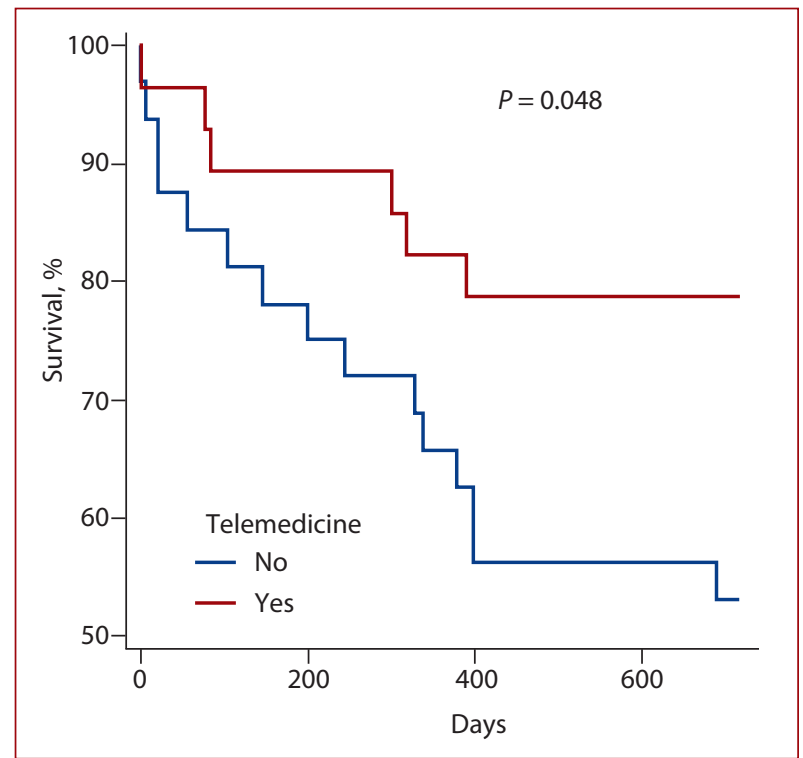

Figure 2. Kaplan-Meier survival curves for survival without the composite endpoint in relation to the use of telemedicine devices

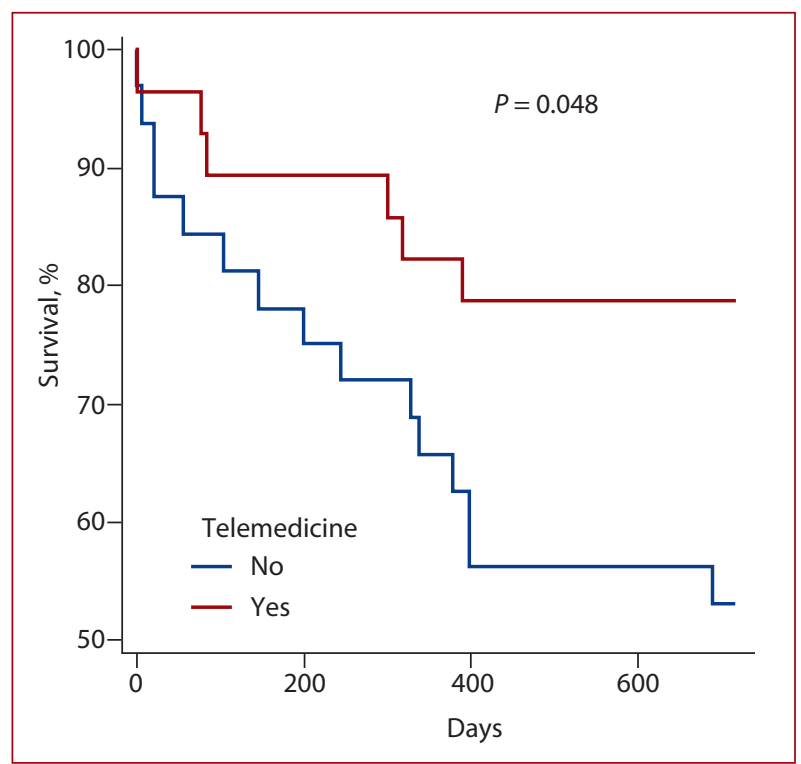

Figure 3. Kaplan-Meier curves for survival without hospitalization for acute heart failure in relation to the use of telemedicine devices

conclusion of our work is the confirmation of the importance of non-invasive telemonitoring for the improvement of long-term prognosis, as well as the improvement of physical performance in the long-term observation. To the best of our knowledge, it is the first clinical trial that used the transmission of so many relevant parameters exclusively in patients with end-stage HF with implanted CRT, from the patient's home to a central hub managed by cardiologists, using non-invasive devices. It differs from the results of a recently published meta-analysis, in which the authors emphasize the important role of nurses who act as medical supervisors in $86 \%$ of programs [6]. One of the first 
multicenter clinical trials in the field of non-invasive monitoring was the Tele-HF study. Also, it did not demonstrate the benefits for patients with HF in terms of reducing the number of hospitalizations and mortality. The daily control was performed with the use of an automatic telephone system, interviews with specialized medical personnel were carried out to a small extent, which, as the authors admitted in the discussion, was a significant limitation. Moreover, in the event of irregularities, contact with the attending physician was recommended, unlike in our study, where the treatment was largely based on the medical team that supervised the study. Also, patients in the TIM-HF study were referred by medical staff supervising telemonitoring to their family medicine doctor or a specialist [7]. Such constructions of telemonitoring solutions, in our opinion, may prolong the time to implement the teletheraphy. That is why our study emphasizes the importance of cooperation between patients and cardiologists who provide online teleconsultation.

Telemonitoring in HF can be implemented with the use of non-invasive or invasive devices, the latter are mainly used in patients with implanted devices. Data from clinical trials indicate that this type of monitoring is useful mainly in improving the quality of life and avoiding unnecessary hospitalization in patients at risk of sudden cardiac death. Telemonitoring reduces the number of visits without affecting the risk of stroke, heart attack, or death [8]. The survival rates were $50 \%$ higher in the group of telemonitored patients in comparison with patients undergoing routine monitoring [9]. The official European Heart Rhythm Association/Heart Rhythm Society (EHRA/HRS) document confirmed the usefulness of telemonitoring of implantable devices in patients with HF, which consists in the earlier detection of clinical disorders due to damage to the device [10]. Invasive telemonitoring in acute and chronic HF received a class IIb recommendation in the 2016 ESC HF guidelines [1].

Despite the recommendations for invasive telemonitoring (with divergent research results: CardioBBEAT, REM-HF, IN-TIME), we used non-invasive telemonitoring, in which the flow of information is more complicated [11, 12]. Firstly, human intervention is needed to deal with non-invasive devices, while invasive monitoring is automated and maintenance-free. Secondly, the patient plays the main role in the non-invasive telemonitoring process, while in the case of invasive monitoring, the patient's involvement is minimal. Thirdly, nurses and GPs are usually involved in non-invasive telemonitoring, while they are not essential for invasive telemonitoring. Fourthly, because of these differences, non-invasive telemonitoring of patients with $\mathrm{HF}$ requires the education and training of patients.

Neither BEAT-HF nor TIM-HF resulted in the reduction of the risk of hospitalization for HF [13]. BEAT-HF researchers reported the real necessity of re-hospitalization in a 6-month observation, while the authors of the TIM-HF study did not demonstrate an interaction between treat- ment effects for the occurrence of the composite endpoint. On the other hand, the results of the meta-analysis by Kitsiou et al. [14] indicate a significant reduction in the risk of mortality and hospitalization for HF in the case of non-invasive telemonitoring. Similar conclusions are provided by the meta-analyses of the studies in the Cochrane Central Register $[14,15]$. Despite the quality of the evidence, data were was obtained with the use of the GRADE methodology, the imprecision of effects varied from moderate to low. That is why, as the authors suggest, further research is necessary. Therefore, the results of the TIM-HF2 multicenter, randomized study, with a highly advanced intervention protocol and defined endpoints, similar to those used in our study, suggested that non-invasive monitoring, when used in a well-defined HF population, could reduce unplanned cardiovascular hospitalizations and all-cause mortality [7].

Interesting results are provided by the analysis of the importance of telemetry for improving physical performance, expressed by improving LVEF or increasing 6MWT distance. In both cases, the use of telemetry proved to be an independent factor in predicting improvement in physical performance, in addition to the widely recognized diameter of the left atrium, the presence of the return wave on the tricuspid valve, or the application of angiotensin-converting-enzyme inhibitors (ACEI) or mineralocorticoid receptor antagonists [16]. Therefore, this is the first report documenting the importance of non-invasive telemetry for increasing 6MWT distance.

Improvement of physical performance was also expressed by a decrease in NT-proBNP value in a remote observation, which reached statistical significance only in the study group. The study by Maric et al. [17] did not confirm the clinical benefit of telemedicine use in reducing NT-proBNP values. In contrast, in the multicenter study by Lazarova et al. [18] on a group of 738 patients, a significant decrease in the value of natriuretic peptides in the longterm observation was reported.

In data presented in the literature, during the COVID-19 outbreak, remote monitoring is of clinical importance in outpatient care, and it seems that extrapolation of our experiences may also be helpful during the pandemic [19-21].

\section{CONCLUSIONS}

The use of non-invasive telemonitoring in a group of patients with CRT therapy improved the prognosis in a 2-year observation period with a significant reduction of hospitalization for severe HF. The use of non-invasive telemetry was an independent predictor of the improvement of left ventricular ejection fraction or the increase of the six-minute walking test distance. It seems that extrapolation of our experiences may be helpful during the outbreak of COVID-19 , and non-invasive telemonitoring should improve medical therapy for patients with HF. Clinical application of non-invasive devices with basic parameters such as weight, BP, saturation, or ECG may substantially improve medical care. 


\section{Limitations}

The main limitation of the study is the small size of the study population and the single-center nature of the study. However, attention should be paid to the heterogeneity of the study group in terms of the incidence of diabetes and in terms of the use of beta-blockers, which were significantly more common in the control group, which is most likely due to the small size of the study group. The exclusion of two patients due to limited access to the network is also a limitation of the study. The study was randomized, but the investigator who performed echocardiography and 6MWT was not blinded. The patients throughout the study were under continuous care by one of the specialists in GCM Electrocardiology Clinic. Therapeutic decisions were based on medical indications and physicians' experience, but without standardization, and finally, there was no comparison of the data.

\section{Supplementary material}

Supplementary material is available at https://journals. viamedica.pl/kardiologia_polska.

\section{Article information}

Conflict of interest: None declared.

Open access: This article is available in open access under Creative Common Attribution-Non-Commercial-No Derivatives 4.0 International (CC BY-NC-ND 4.0) license, allowing to download articles and share them with others as long as they credit the authors and the publisher, but without permission to change them in any way or use them commercially. For commercial use, please contact the journal office at kardiologiapolska@ptkardio.pl.

\section{REFERENCES}

1. Ponikowski P, Voors AA, Anker SD, et al. 2016 ESC Guidelines for the diagnosis and treatment of acute and chronic heart failure: The Task Force for the diagnosis and treatment of acute and chronic heart failure of the European Society of Cardiology (ESC). Developed with the special contribution of the Heart Failure Association (HFA) of the ESC. Eur J Heart Fail. 2016;37(27):2129-2200, doi: 10.1093/eurheartj/ehw128, indexed in Pubmed: 27206819.

2. Brignole M, Auricchio A, Baron-Esquivias G, et al. 2013 ESC Guidelines on cardiac pacing and cardiac resynchronization therapy: The Task Force on cardiac pacing and resynchronization therapy of the European Society of Cardiology (ESC). Developed in collaboration with the European Heart Rhythm Association (EHRA). Europace. 2013; 15(8): 1070-1118, doi: 10.1093/europace/eut206, indexed in Pubmed: 23801827.

3. Penn J, Goldenberg I, Moss AJ, et al. Cardiac-resynchronization therapy for the prevention of heart-failure events. N Engl J Med. 2009; 361(14): 1329-1338, doi: 10.1056/NEJMoa0906431, indexed in Pubmed: 19723701.

4. Chaudhry SI, Phillips CO, Stewart SS, et al. Telemonitoring for patients with chronic heart failure: a systematic review. J Card Fail. 2007; 13(1): 56-62, doi: 10.1016/j.cardfail.2006.09.001, indexed in Pubmed: 17339004.

5. Cowie $\mathrm{M}, \mathrm{BAX}$ J, Bruining $\mathrm{N}$, et al. et al.. e-Health: a position statement of the European Society of Cardiology. Eur Heart J. 2016; 37(1): 63-66, doi: 10.1093/eurheartj/ehv416, indexed in Pubmed: 26303835.

6. Farnia T, Jaulent MC, Steichen O. Evaluation criteria of noninvasive telemonitoring for patients with heart failure: systematic review. J Med Internet Res. 2018; 20(1):e16, doi: 10.2196/jmir.7873, indexed in Pubmed: 29339348.
7. Koehler F, Koehler K, Deckwart O, et al. Telemedical Interventional Management in Heart Failure II (TIM-HF2), a randomised, controlled trial investigating the impact of telemedicine on unplanned cardiovascular hospitalisations and mortality in heart failure patients: study design and description of the intervention. Eur J Heart Fail. 2018; 20(10): 1485-1493, doi: 10.1002/ejhf.1300, indexed in Pubmed: 30230666.

8. Varma N, Michalski J, Stambler B, et al. Superiority of automatic remote monitoring compared with in-person evaluation for scheduled ICD follow-up in the TRUST trial - testing execution of the recommendations. Eur Heart J. 2014; 35(20): 1345-1352, doi: 10.1093/eurheartj/ehu066, indexed in Pubmed: 24595864.

9. Saxon LA, Hayes DL, Gilliam FR, et al. Long-term outcome after ICD and CRT implantation and influence of remote device follow-up: the ALTITUDE survival study. Circulation. 2010; 122(23): 2359-2367, doi: 10.1161/CIRCULATIONAHA.110.960633, indexed in Pubmed: 21098452.

10. Daubert JC, Saxon L, Adamson PB, et al. 2012 EHRA/HRS expert consensus statement on cardiac resynchronization therapy in heart failure: implant and follow-up recommendations and management. Heart Rhythm. 2012; 9(9): 1524-1576, doi: 10.1016/j.hrthm.2012.07.025, indexed in Pubmed: 22939223.

11. Hofmann R, Völler H, Nagels K, et al. First outline and baseline data of a randomized, controlled multicenter trial to evaluate the health economic impact of home telemonitoring in chronic heart failure - CardioBBEAT.Trials. 2015; 16: 343, doi: 10.1186/s13063-015-0886-8, indexed in Pubmed: 26259568.

12. Morgan JM, Kitt S, Gill J, et al. Remote management of heart failure using implantable electronic devices. Eur Heart J. 2017; 38(30): 2352-2360, doi: 10.1093/eurheartj/ehx227, indexed in Pubmed: 28575235.

13. Koehler $F$, Winkler $S$, Schieber $M$, et al. Telemedicine in heart failure: pre-specified and exploratory subgroup analyses from the TIM-HF trial. Int J Cardiol. 2012; 161(3): 143-150, doi: 10.1016/j.ijcard.2011.09.007, indexed in Pubmed: 21982700.

14. Kitsiou S, Paré G, Jaana M. Effects of home telemonitoring interventions on patients with chronic heart failure: an overview of systematic reviews. J Med Internet Res. 2015; 17(3): e63, doi: 10.2196/jmir.4174, indexed in Pubmed: 25768664.

15. Inglis SC, ClarkRA, Dierckx R, et al. Telemonitoring or structured telephone support programmes for patients with chronic heart failure: systematic review and meta-analysis. BMJ. 2007; 334(7600): 942-1040, doi: 10.1136/bmj.39156.536968.55, indexed in Pubmed: 17426062.

16. Nagueh S, Appleton C, Gillebert T, et al. et al.. Recommendations for the evaluation of left ventricular diastolic function by echocardiography. J Am Soc Echocardiogr. 2009; 22(2): 107-133, doi: 10.1016/j.echo.2008.11.023, indexed in Pubmed: 19187853.

17. Maric B, Kaan A, Araki Y, et al. The use of the internet to remotely monitor patients with heart failure. Telemed J E Health. 2010; 16(1): 26-33, doi: 10.1089/tmj.2009.0094, indexed in Pubmed: 20070163.

18. Lazarova M, Lazar D, Malek F, et al. Heart failure disease management program, its contribution to established pharmacotherapy and long-term prognosis in real clinical practice - retrospective data analysis. Biomed Pap Med Fac Univ Palacky Olomouc Czech Repub. 2019; 163(4): 318-323, doi: 10.5507/bp.2018.059, indexed in Pubmed: 30305762.

19. Tajstra M, Wojtaszczyk A, Sterliński M, et al. Patients with heart failure and an implanted cardioverter-defibrillator during the coronavirus disease 2019 pandemic: insights from a multicenter registry in Poland. Kardiol Pol. 2021; 79(5): 562-565, doi: 10.33963/KP.15918, indexed in Pubmed: 34125930.

20. Budrejko S, Kempa M, Królak T, et al. Procedural volume of cardiac electrotherapy procedures does not have to be reduced during COVID-19 pandemic — one year analysis from a tertiary Polish center. Kardiol Pol. 2021; 79(7-8): 848-850, doi: 10.33963/kp.a2021.0001, indexed in Pubmed: 34013513.

21. Wranicz JK, Drożdż J, Różycka-Kosmalska M, et al. Impact of the COVID-19 pandemic on the performance of selected cardiac electrotherapy and electrophysiology procedures. Kardiol Pol. 2021; 79(4): 452-454, doi: 10.33963/KP.15884, indexed in Pubmed: 33754584. 